Case Report

\title{
Left Ventricular Noncompaction: A Rare Case of Nonischemic Cardiomyopathy
}

\author{
Julia Tian, ${ }^{1}$ Asif Uddin $(1),{ }^{1}$ and Philippe Akhrass ${ }^{2}$ \\ ${ }^{1}$ Department of Medicine, Northwell Health at Staten Island University Hospital, Staten Island, NY 10305, USA \\ ${ }^{2}$ Department of Electrophysiology, Northwell Health at Staten Island University Hospital, Staten Island, NY 10305, USA
}

Correspondence should be addressed to Asif Uddin; asif1uddin@gmail.com

Received 6 March 2019; Accepted 4 July 2019; Published 8 August 2019

Academic Editor: Magnus Baumhäkel

Copyright ( 2019 Julia Tian et al. This is an open access article distributed under the Creative Commons Attribution License, which permits unrestricted use, distribution, and reproduction in any medium, provided the original work is properly cited.

\begin{abstract}
Isolated left ventricular noncompaction (LVNC) is a rare form of cardiomyopathy that is characterized by deep intertrabecular recesses and abnormal trabeculations that can be observed on transthoracic echocardiogram (TTE) or cardiac MRI (CMR) studies. Our case describes a 41-year-old male who presented with exertional chest pain and was discovered to have significantly reduced left ventricular ejection fraction (LVEF) which was nonischemic in etiology as confirmed by cardiac catheterization. Subsequent evaluation with CMR imaging revealed noncompaction of the left ventricle. The patient received defibrillation and lifelong anticoagulation given his elevated risk of sudden cardiac death (SCD). This case highlights the importance of considering unconventional etiologies of cardiomyopathy when investigating new-onset heart failure as well as the necessity of life-saving measures such as anticoagulation and defibrillator implantation in view of arrhythmogenic structural heart diseases.
\end{abstract}

\section{Introduction}

Left ventricular noncompaction (LVNC) is an uncommon cardiomyopathy estimated to occur between 0.014 and 1.3 percent in the general population. It is characterized by deep intertrabecular recesses and abnormal trabeculations popularly believed to result from a failure of a phase of cardiac development during which the normal myocardium compacts inward. However, it can also involve abnormalities during ventricular wall formation or remodeling which can be transient or permanent $[1,2]$.

Clinical manifestations vary widely and can range from asymptomatic disease to symptoms of heart failure, left ventricular systolic dysfunction, thromboembolism, WolffParkinson-White (WPW) syndrome, atrial and ventricular arrhythmias, bundle branch blocks, and sudden cardiac death. The most common manifestations are heart failure and ventricular systolic dysfunction.

There is currently no universally-accepted criteria for classifying and diagnosing LVNC. Depending on the organization, it may be classified as a genetic cardiomyopathy or as an unclassified cardiomyopathy [3,4]. Diagnosis of LVNC can be made using imaging studies such as echocardiography, computed tomography, or CMR. Similar to the clinical criteria, no standard imaging criteria currently exist. The Jenni, Chin, and Stollberger criteria emphasize different components of the echocardiogram $[5,6]$. A summary of all three would involve evidence of a two-layered structure (thin compacted epicardial layer and thick endocardial layer) with a noncompacted to compacted ratio of greater than 2 (greater than 2.3 on CMR), an epicardial-to-trabeculation trough to epicardial-to-trabeculation peak ratio of less than 0.5 , and more than three trabeculations from the ventricular wall (not sensitive for CMR) $[7,8]$.

\section{Case Presentation}

The patient was a 41-year-old male with a past medical history of unspecified congenital heart disease, hypothyroidism, and hypogonadism (taking intramuscular testosterone injections twice weekly), who presented with a chief complaint of chest pain during exertion of 1-month duration. The pain 


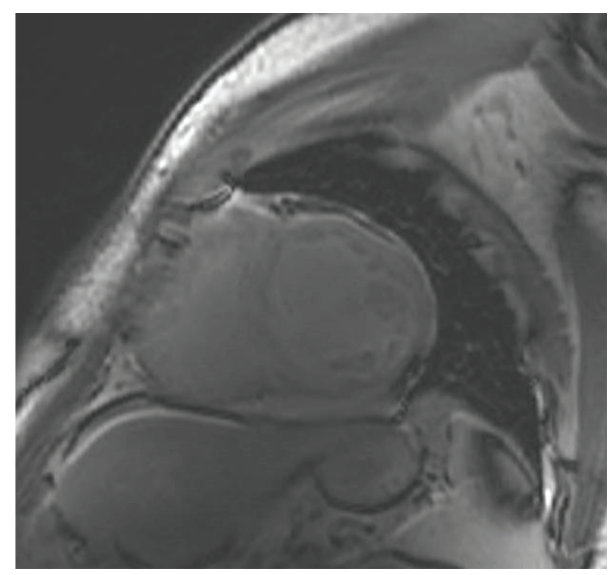

Figure 1: Short axis view of cardiac magnetic resonance (CMR) reveals a noncompact-to-compact myocardium ratio of greater than 2.3 .

was described as 10 out of 10 in intensity, burning with a band-like tightness in nature, and located in the epigastric area with radiation toward the substernal region. His initial cardiac enzymes were significant for a troponin level of $0.62 \mathrm{ng} / \mathrm{mL}$. His B-type natriuretic peptide (BNP) level was 1,190. An electrocardiogram (ECG) revealed a sinus rhythm with right superior axis deviation, biatrial enlargement, and right ventricular hypertrophy. Further evaluation with a TTE revealed that the patient had a LVEF of $20-29 \%$ with a markedly dilated left atrium and left ventricle. Subsequently, a cardiac catheterization did not reveal evidence of obstructive coronary artery disease. Given the trivial history of congenital heart disease and nonischemic cardiomyopathy, a cardiac MRI was then conducted and revealed a noncompacted to compacted myocardium ratio of greater than 2.3 in the left ventricle, global hypokinesis with severely depressed left and right ventricle ejection fractions of $27 \%$ and $26 \%$, biatrial enlargement, and no evidence of a left ventricular thrombus (Figures 1 and 2). His hospital course was complicated by an episode of nonsustained ventricular tachycardia, which given his LVNC and low ejection fraction, placed him at an elevated risk for SCD. The patient subsequently received an internal cardiac defibrillator (ICD) and was discharged home with aspirin, beta blocker, angiotensin-receptor blocker, and anticoagulation therapy with warfarin.

\section{Case Discussion}

In summary, LVNC is an uncommon and controversial cause of cardiomyopathy. It is becoming a popular topic in the field of cardiology due to its multiple possible etiologies, pathogenesis, diagnostic criteria, and clinical course. Due to its highly variable manifestations, the management of this condition should be individualized. Patients with evidence of heart failure should receive guideline-directed medical therapy (GDMT). Some literature supports the use of anticoagulation in patients with LVEF less than 40\%, a history of atrial fibrillation, or a prior cardioembolic event

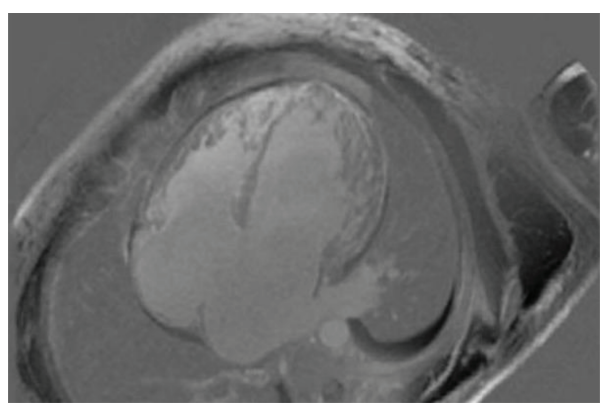

FIgURE 2: Four-chamber view of cardiac magnetic resonance (CMR) reveals dilation of all chambers of the heart.

[9]. All patients should also receive family and genetic counseling. As an uncommon disease with a multitude of life-threatening complications, it is imperative to perform a thorough cardiac evaluation in cases of new-onset heart failure with or without arrhythmia. In addition, further research and collaboration from major organizations are necessary to create a standardized criteria for the diagnosis and management of this disorder.

\section{Conflicts of Interest}

The authors declare that there is no conflict of interest regarding the publication of this paper.

\section{References}

[1] H. Chen, W. Zhang, D. Li, T. M. Cordes, R. Mark Payne, and W. Shou, "Analysis of ventricular hypertrabeculation and noncompaction using genetically engineered mouse models," Pediatric Cardiology, vol. 30, no. 5, pp. 626-634, 2009.

[2] E. Arbustini, F. Weidemann, and J. L. Hall, "Left ventricular noncompaction: a distinct cardiomyopathy or a trait shared by different cardiac diseases?," Journal of the American College of Cardiology, vol. 64, no. 17, pp. 1840-1850, 2014.

[3] B. J. Maron, J. A. Towbin, G. Thiene et al., "Contemporary definitions and classification of the cardiomyopathies: an American Heart Association scientific statement from the Council on Clinical Cardiology, Heart Failure and Transplantation Committee; Quality of Care and Outcomes Research and Functional Genomics and Translational Biology Interdisciplinary Working Groups; and Council on Epidemiology and Prevention," Circulation, vol. 113, no. 14, pp. 1807-1816, 2006.

[4] P. Elliott, B. Andersson, E. Arbustini et al., "Classification of the cardiomyopathies: a position statement from the European Society of Cardiology Working Group on Myocardial and Pericardial Diseases," European Heart Journal, vol. 29, no. 2, pp. 270-276, 2008.

[5] B. S. Frischknecht, C. H. Attenhofer Jost, E. N. Oechslin et al., "Validation of noncompaction criteria in dilated cardiomyopathy, and valvular and hypertensive heart disease," Journal of the American Society of Echocardiography, vol. 18, no. 8, pp. 865$872,2005$.

[6] T. K. Chin, J. K. Perloff, R. G. Williams, K. Jue, and R. Mohrmann, "Isolated noncompaction of left ventricular myocardium. A study of eight cases," Circulation, vol. 82, no. 2, pp. 507-513, 1990. 
[7] G. Fazio, G. Novo, L. D'angelo et al., "Magnetic resonance in isolated noncompaction of the ventricular myocardium," International Journal of Cardiology, vol. 140, no. 3, pp. 367-369, 2010.

[8] C. Stöllberger, W. Kopsa, R. Tscherney, and J. Finsterer, "Diagnosing left ventricular noncompaction by echocardiography and cardiac magnetic resonance imaging and its dependency on neuromuscular disorders," Clinical Cardiology, vol. 31, no. 8, pp. 383-387, 2008.

[9] K. Kido and M. Guglin, “Anticoagulation Therapy in Specific Cardiomyopathies: Isolated Left Ventricular Noncompaction and Peripartum Cardiomyopathy," Journal of Cardiovascular Pharmacology and Therapeutics, vol. 24, no. 1, pp. 31-36, 2019. 


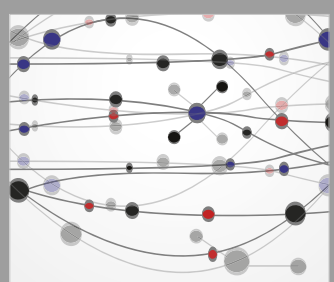

The Scientific World Journal
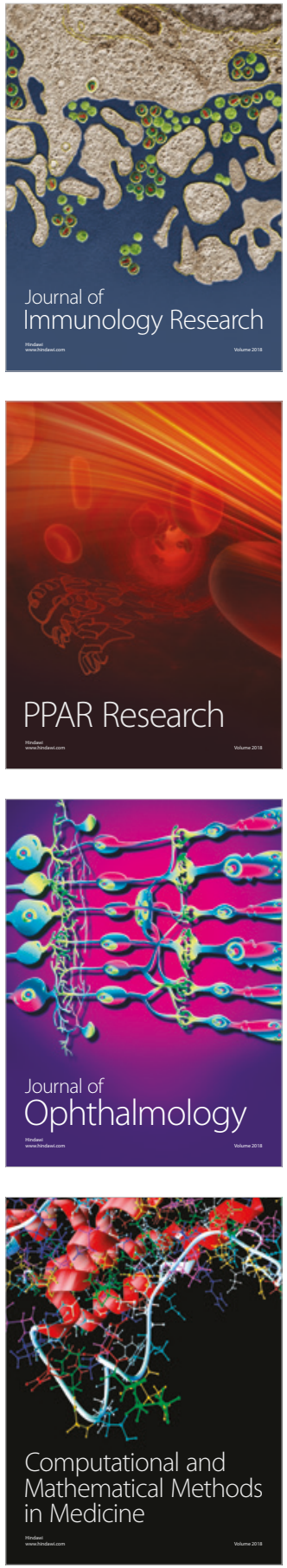

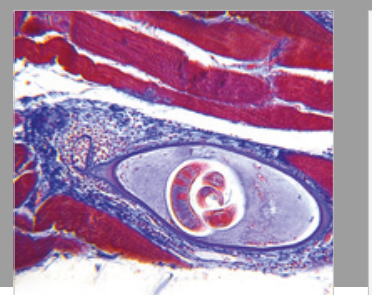

Gastroenterology Research and Practice

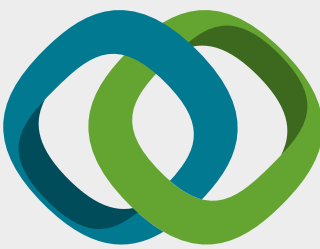

\section{Hindawi}

Submit your manuscripts at

www.hindawi.com
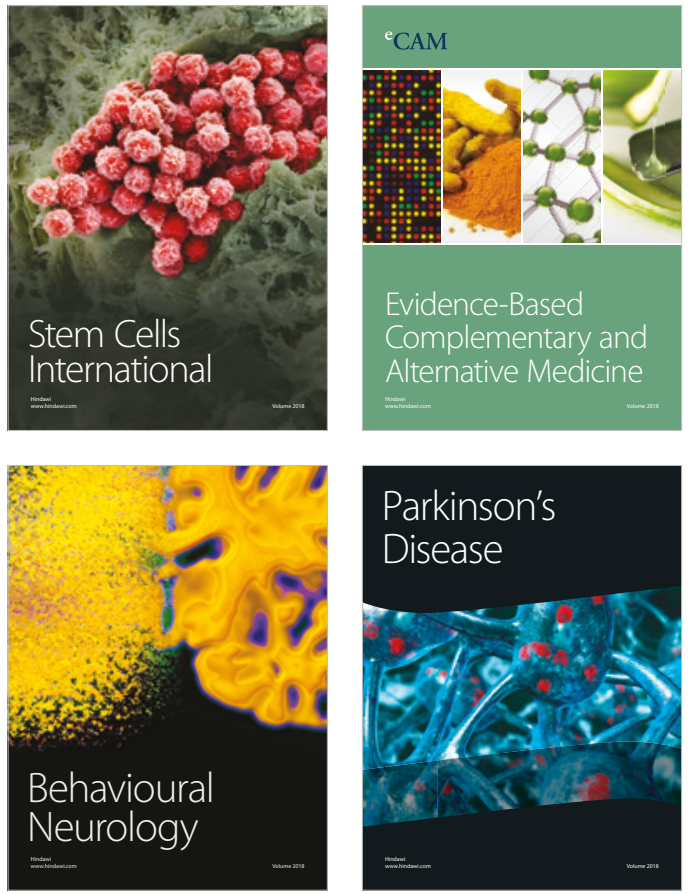

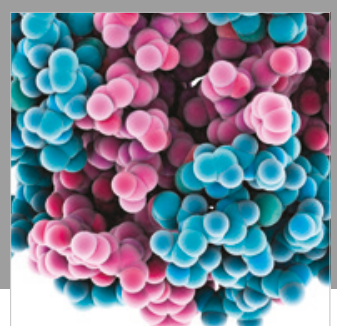

ournal of

Diabetes Research

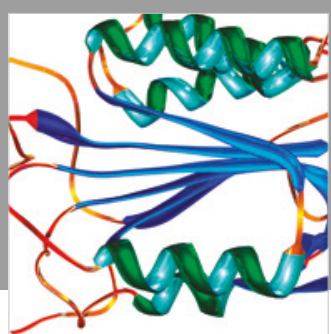

Disease Markers
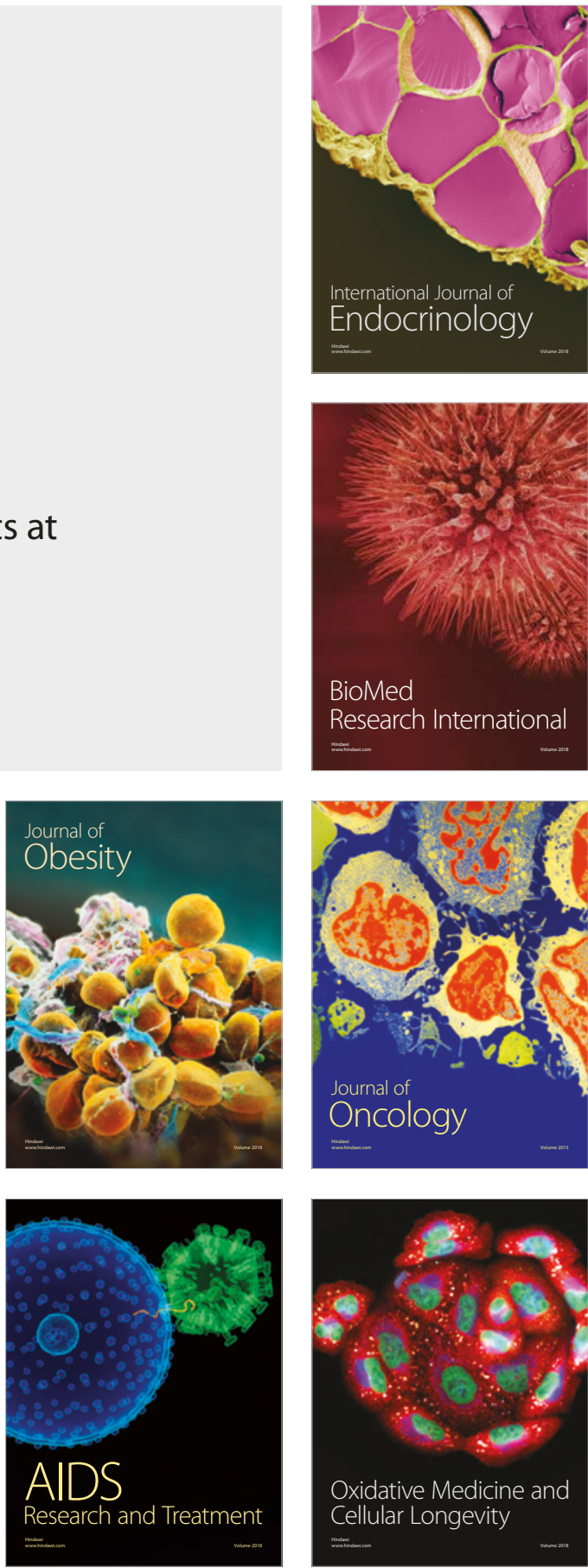